ÉGYPTE

monde arabe

\section{Égypte/Monde arabe}

14 | 1993

Dits et écrits, mémoires et rites

\title{
Epopée et sociologie : le cas de l'Égypte
}

Ali Fahmi

\section{Q OpenEdition}

Journals

Édition électronique

URL : https://journals.openedition.org/ema/565

DOI : 10.4000/ema.565

ISSN : 2090-7273

\section{Éditeur}

CEDEJ - Centre d'études et de documentation économiques juridiques et sociales

\section{Édition imprimée}

Date de publication : 30 juin 1993

Pagination : 61-66

ISSN : 1110-5097

\section{Référence électronique}

Ali Fahmi, «Epopée et sociologie : le cas de l'Égypte », Égypte/Monde arabe [En ligne], 14| 1993, mis en ligne le 08 juillet 2008, consulté le 07 juillet 2022. URL : http://journals.openedition.org/ema/565 ; DOI : https://doi.org/10.4000/ema.565

Ce document a été généré automatiquement le 7 juillet 2022

Tous droits réservés 


\title{
Epopée et sociologie : le cas de l'Égypte
}

\author{
Ali Fahmi
}

1 L'épopée ${ }^{1}$ est un de ces arts populaires qui s'épanouissent généralement dans les sociétés où récit et tradition orale tiennent une place importante, soit qu'on goûte particulièrement ce mode d'expression, soit que l'analphabétisme y sévisse plus ou moins, ou encore pour ces deux raisons à la fois. Dans le cas de la Nation arabe, dont l'Égypte, c'est plutôt cette dernière hypothèse qui prévaut depuis le temps de 'Ukaz et des célèbres mu'allaqât ${ }^{2}$.

2 Né au sein du peuple et transmis de génération en génération, le récit populaire prend naissance chez les gens de modeste extraction : ils en sont tout à la fois les auteurs, les conteurs et les auditeurs. Ils l'inventent en toute spontanéité pour ensuite le transmettre, puis l'écouter et le réécouter inlassablement avec toutes les modifications, suppressions ou ajouts intervenus au fil du temps et au gré des fluctuations de la sensibilité populaire.

3 Nous avons choisi de limiter à notre étude à des récits purement égyptiens, tant par leur origine et le cadre de leur diffusion que par les événements relatés et les personnages mis en scène. A notre avis, en effet, en dépit d'une culture générale imprégnée d'arabité, le peuple égyptien a son identité propre, sa spécificité, repérables au travers de son autonomie géopolitique et de l'histoire de sa civilisation ${ }^{3}$.

4 Nous évoquerons donc, au début de ce bref aperçu, les traits marquants de l'identité égyptienne, pour présenter ensuite certaines hypothèses relatives aux récits populaires égyptiens - plus particulièrement L'Epopée d'al-Zaher Baybars et L'Epopée de 'Ali al-Zaybaq - ainsi que certaines constantes sociales et culturelles observées précisément dans ces deux récits. Enfin, nous nous demanderons quel profit la sociologie égyptienne, plus particulièrement l'histoire sociale, peut tirer du récit populaire.

De l'identité égyptienne

5 La société égyptienne a vécu pendant des siècles sur les rives d'un fleuve sujet à des crues régulières. Si la crue annuelle avait lieu à date plus ou moins fixe, il n'en était pas 
de même de la distribution de l'eau qui, elle, variait d'une saison à l'autre. Il était donc essentiel qu'un pouvoir central fort en assurât le contrôle et la maitrise. L'existence d'un tel pouvoir constitue une caractéristique non négligeable. Il importe également qu'il soit doté d'une force répressive - pour ne pas dire tyrannique - et qu'il dispose de moyens de contrôle efficaces. La longue histoire de l'Égypte en témoigne.

6 A l'exception d'une minorité de Bédouins, les Egyptiens étaient tous concentrés dans l'étroite vallée du Nil, tandis que dans les vastes étendues alentour, une mort certaine attendait celui qui, voulant fuir l'autorité, croyait pouvoir y trouver asile. Voilà pourquoi, pour affronter le tyran, le peuple égyptien eut recours à des stratagèmes d'autodéfense multiples, originaux et efficaces : anecdotes, rumeurs, proverbes.... Il eut également recours à l'épopée, support de projection de ses rêves, de sa patience historique, de ses espoirs déçus. Il fit enfin, du temps et de la mort inéluctables, ses alliés.

7 Ces traits, ces constantes, ont été relevés par les historiens et les voyageurs. Hérodote d'abord, avant l'ère chrétienne. Ensuite, à l'époque arabo-musulmane, depuis Ibn 'Abd al-Hakam et les illustres futuhât $t^{5}$ jusqu'à 'Ali Moubarak à la fin du xix ${ }^{e}$ siècle, en passant par toute une constellation d'historiens et chroniqueurs comme al-Maqrizi, Ibn lyas et al-Jabarti.

8 Soulignons toutefois que si les récits des historiens et des voyageurs ont rassemblé une profusion de traits caractéristiques, ils se sont contentés d'une recension superficielle sans essayer véritablement de comprendre, $d$ 'analyser, $d$ 'interpréter les stratagèmes de défense du peuple égyptien'.

Nous tenterons donc ici une nouvelle lecture d'un de ces stratagèmes, l'épopée.

Réinventer l'histoire

10 Nous poserons ici comme hypothèse de base que le récit populaire en Égypte permettait de reconstituer les événements et faits historiques indépendamment de ce qu'en montrait la réalité objective. Restructuration représentative, à notre avis, d'une volonté collective d'insurrection contre le pouvoir absolu, le récit populaire est l'occasion d'exprimer une révolte refoulée, latente mais prête à exploser à tout moment. Dans cette hypothèse, l'épopée n'est pas seulement pour le peuple une manière de défoulement mais encore - voire davantage - un moyen d'affronter des événements qui contrecarrent ses aspirations nationales : on crée de toutes pièces des « héros populaires » et on relate, à travers leurs exploits, des événements imaginaires se déroulant sur une scène jusqu'alors occupée par des dirigeants omnipotents.

$11 \mathrm{Si}$, à la lumière de ce qui précède, nous comparons les événements tels qu'ils sont relatés dans le récit et les faits réels, nous constatons en effet que le récit, loin d'être conforme à la réalité, reflète une forme particulière de réaction populaire aux agissements du pouvoir, tentative de reconstruction hypothétique de l'histoire, voire même d'élaboration de scénarios futuristes qui pourront servir aux générations à venir et leur inculquent l'esprit de résistance. Le récit propose des leçons destinées à empêcher que se reproduisent les mêmes événements, l'imaginaire populaire se substituant à une réalité historique impitoyable.

On peut se demander pourquoi les Egyptiens éprouvent tant de sympathie envers le personnage d'al-Zâher Baybars, héros épique d'un des récits les plus célèbres. Cette époque de l'histoire de l'Égypte a en effet connu des sultans tout aussi prestigieux que Baybars et qui, comme Salah al-Din al-Ayûbi, étaient dotés d'une personnalité hors du 
commun. Cependant, le récit populaire accorde à des personnages tels qu'al-Saleh Negm al-Din Ayûb, dernier sultan de la période ayûbide - celui qui avait élevé Baybars - une place plus importante qu'au fameux Salah al-Din al-Ayûbi, qui a joué le rôle le plus prestigieux de l'histoire des Croisades. Cela pourrait s'expliquer par le fait que le sentiment populaire ne tolère aucune complaisance vis-à-vis des adversaires de la nation. Or, selon Ibn al-Asir, "Salah al-Din était par trop laxiste avec ses adversaires ». La conscience populaire ne lui a pas pardonné ce « laxisme » qui pourtant, à notre sens, relève davantage de la tolérance religieuse et de l'éthique chevaleresque que du renoncement aux droits nationaux.

Mettons en parallèle l'image de Salah al-Din et celle de Baybars, le premier à avoir combattu les Mongols et rétabli le califat abbasside. Acte dont le mérite a été reconnu par Ibn lyas, exploit qui a profondément influencé le peuple égyptien et a donné à son auteur une véritable légitimité, en dépit de ses origines d'esclave mamelouk et bien qu'il se fût emparé par la force du trône des Ayûbides. Un tel parallèle nous fait comprendre pourquoi le peuple égyptien a immortalisé Baybars sans s'attarder sur l'histoire glorieuse de Salah al-Din et son rôle de défenseur de l'islam et des musulmans ${ }^{7}$ : ce qui, selon nous, a enflammé la sensibilité populaire et immortalisé Baybars, c'est la légitimité sur laquelle est fondée l'épopée. Nous avancerons même que, tout au long de l'histoire et jusqu'à nos jours, la légitimité a été et continue à être un critère quasi sacré dans l'épopée égyptienne ${ }^{8}$. La conscience populaire célèbre bien évidemment les triomphes militaires contre les ennemis de la nation et la défaite de ses adversaires, mais elle célèbre d'une manière toute particulière la question de la légitimité et la participation populaire au pouvoir que cette légitimité peut entraîner.

Cette question de légitimité .et de participation au pouvoir, jamais ou rarement concrétisée dans la réalité, a toujours constitué le rêve du peuple égyptien tout au long de son histoire. Aussi, lorsqu'il s'agit de réécrire celle-ci à travers les épopées, la conscience populaire insiste outrancièrement sur ces deux aspects. L'épopée zahérienne ne met-elle pas en scène un personnage fictif présenté comme un fils du peuple, Osta 'Uthman Ibn al-Hebali, associé d'al-Zaher au pouvoir, ainsi qu'un autre héros, Gamal al-Din Chiha, non pas Egyptien mais Palestinien de Gaza et présenté comme collaborateur militaire d'al-Zaher Baybars? Le récit lui attribue la vivacité d'esprit qui caractérise les Egyptiens, de sorte que « les astuces de Chiha » sont passées dans les proverbes.

15 Un autre rédt, L'Epopée de 'Ali al-Zaybaq', vient confirmer ce point de vue en relatant une succession interminable de luttes et d'actions habiles et pleines d'esprit menées par le héros. Pour finir, le sultan propose à 'Ali al-Zaybaq de participer au pouvoir aux côtés du tyran Salah al-Kalbi. Une fois réalisé ce rêve, 'Ali Al-Zaybaq déclare : «Désormais, je m'opposerai à l'injustice de Salah al-Kalbi et préviendrai le mal qu'il cause; je répandrai la justice parmi les hommes et rendrai leurs droits à ceux que sa violence et sa perfidie en ont privé. »

16 Ce personnage créé par l'imaginaire populaire concrétise une fois encore le rêve du «fils du peuple » de prendre part activement, après avoir lutté contre les symboles de l'oppression, au gouvernement de son pays. Nous constatons par ailleurs que dans les épopées, cette ambition ne va pas jusqu'à vouloir s'emparer du pouvoir. Il s'agit seulement d'y participer. Le peuple égyptien, malgré toutes les épreuves qu'il a endurées, fait preuve d'une sorte de modestie réaliste : il aspire à partager le pouvoir sans pour autant vouloir en écarter ses représentants. Partage du pouvoir qui vise à le 
réformer, à mettre un terme à la corruption, à rétablir la justice. Rêve qui repose sur "l'art du possible», dans un contexte historique bien précis. Nous conclurons de ce qui précède qu'il nous faut ajouter, aux caractéristiques nationales énoncées plus haut, une dimension essentielle : une sagesse mesurée puisée dans un imaginaire débridé. Le rêve populaire de partage du pouvoir, de « légitimité ", est un rêve empreint de ténacité, de maturité et de pondération.

Revenons à l'épopée d'al-Zaher Baybars. Nous y retrouvons un thème récurrent, celui de la corruption en Égypte. Il est donc tout naturel que le héros soit particulièrement représentatif de la société, donc informé des manifestations secrètes de la corruption surtout si, comme dans le cas de Baybars, le personnage principal de l'épopée est d'origine étrangère et qu'il veut, lui aussi, épurer le pays d'une corruption croissante et mener une action réformiste de grande envergure ${ }^{10}$.

Le personnage central de cette épopée, Osta 'Uthman Ibn al-Hebali, est fictif, inventé par la conscience populaire. C'est un Cairote du célèbre et toujours vivant quartier populaire d'al-Husayniyya. Ce héros épique, que le pouvoir n'a jamais réussi à vaincre, n'est pas un hors-la-loi mais seulement un rebelle qui, dans toute l'Égypte, n'avait de respect que pour deux personnes, le sultan aI-SaIeh Negm al-Din Ayûb, représentant de la plus haute légitimité, et le juge 'Izz al-Din 'Abd al-Salâm, symbole de la science religieuse et de l'honorable charîa.

Notons encore que l'imaginaire égyptien tente de conférer au héros qu'il a créé une certaine légitimité, en faisant en sorte que, grâce à l'intercession de Sayyida Nefissa, fille de Hasan al-Anwar et descendante du Prophète - figure que vénèrent particulièrement les Egyptiens - une sorte de conciliation s'instaure entre le héros et Baybars avant l'accès de ce dernier au pouvoir. Ce qui est surprenant, c'est que Sayyida Nefissa mourut environ huit siècles avant l'époque où se déroule l'action...

Nous nous demanderons pour conclure dans quelle mesure l'analyse de l'épopée peut éclairer la compréhension de l'histoire sociale égyptienne. Nous soutenons pour notre part que, sans une compréhension profonde de cette histoire, il est impossible de saisir la réalité sociale d'aujourd'hui.

21 Une approche attentive et approfondie, qui tenterait de lire entre les lignes, peut donc être d'une grande utilité dans l'étude de l'histoire sociale, à condition que le lecteur n'y cherche pas des faits historiques mais tente plutôt de discerner ce qui se cache derrière le récit, notamment les stratagèmes de défense qu'il recèle. Allons même plus loin et suggérons au lecteur une approche peu commune : considérer les faits racontés dans l'épopée comme reflétant - dans une certaine mesure - l'inverse de ce qui s'est produit dans la réalité... Car l'épopée - hormis sa valeur littéraire - n'a pas pour but de refléter le réel mais de maintenir l'esprit de résistance et de préfigurer un meilleur avenir.

Traduit de l'arabe par Samia Rizq 


\section{NOTES}

1. CNRSC : Centre national de recherches sociales et criminologiques, Le Caire. A.

Fahmi, sociologue, est conseiller de cette institution.

2. Mu'allaqât : poèmes antéislamiques célèbres (ndlr).

3. Hamdan Gamal, Chakhsiat Masr [L'Identité de l'Egypte], Dar al-Hilâl, Le Caire, 1969.

4. Fahmi Ali, Farag Safwat, Hiwâr bayn manhajayn [Confrontation de deux méthodologies], Madbouli, Le Caire, 1981.

5. Futuhât: donations religieuses, offrandes (ndlr).

6. Fahmi Ali, Ai-Tahâyul 'ala ma'âyich fi Misr al-mahrûsa. Dirâsa fi âliyyât al-ta'âmul ma'a alfaqr wa-l-qahr [Des moyens de contourner les difficultés de la vie quotidienne au Caire. Etude sur les moyens de lutte contre la pauvreté et l'oppression], Conférence de l'Association arabe de sociologie, Madrid, 1990.

7. Al-Naggar Muhammad Ragab, Al-bataI fi'l-malâmih al-cha'biyya al-'arabiyya. Qadayâhu wa malâmihuhu al-fanniyya [Le héros dans l'épopée arabe], Thèse de doctorat (non éditée), Faculté des Lettres, Université du Caire, 1967.

8. Id., pp. 282-287.

9. Khorchid Faruq, Ali al-Zaybaq. 2e partie, Dâr al-Hilâl, Le Caire, septembre 1967.

10. Fahmi Ali, Misr wa zâhirat tamarrud al-jund [L'Egypte et la mutinerie des conscrits], Revue Al-Tadammun, Londres, mars 1986.

INDEX

Mots-clés : littérature, identité, épopée, pouvoir

\section{AUTEUR}

ALI FAHMI

CNRSC 